\title{
Review of: "Fate transitions in Drosophila neural lineages: a single cell road map to mature neurons"
}

\author{
Himanshu Pawankumar Gupta
}

Potential competing interests: The author(s) declared that no potential competing interests exist.

The manuscript by Marques et al. presents the transcriptomic changes during the transition of neuroblasts into mature neurons in a short time window. The work highlighted the power of single-cell RNA sequencing in resolving the intermediate immature GMCs, which were difficult to study using traditional methods. Overall the manuscript is good.

The following are my concerns/suggestions:

- In figure 4, whether the authors have used the same color code for representing cell type in the cell trajectory analysis and clusters in the heat map? Mentioning this information in the results or figure legends could be helpful for the readers.

- Recently, a study from Lacin et al., 2019 showed ChAT transcripts were detected in many glutamatergic and GABAergic neurons of CNS, but ChAT transcripts never get translated. While in the present study, using a ChAT as a case study, the author claims that ChAT transcripts were detected in the newly born neurons while its translation occurs at the time of neuronal maturation. Therefore, examining transcripts and protein levels for few other neurotransmitter genes will support the findings that there is pause between transcription and translation depending on the age and stage of neurons. 